\title{
An acutely angled high takeoff left main coronary artery in an aortic root and proximal arch aneurysm
}

\author{
Kyung Hwa Kim, MD, PhD, Jeonju, Republic of Korea
}

\footnotetext{
From the Department of Thoracic and Cardiovascular Surgery, Chonbuk National University Medical School, Chonbuk National University Hospital, Jeonju, Republic of Korea; and the Research Institute of Clinical Medicine of Chonbuk National University and Biomedical Research Institute of Chonbuk National University Hospital, Jeonju, Republic of Korea.

Funding from the Research Institute of Clinical Medicine of Chonbuk National University and Biomedical Research Institute of Chonbuk National University Hospital is gratefully acknowledged.

Disclosures: Author has nothing to disclose with regard to commercial support.

Received for publication Dec 8, 2018; revisions received Dec 28, 2018; accepted for publication Jan 2, 2019; available ahead of print Feb 7, 2019.

Address for reprints: Kyung Hwa Kim, MD, PhD, Department of Thoracic and Cardiovascular Surgery, Chonbuk National University Medical School, Chonbuk National University Hospital, Research Institute of Clinical Medicine of Chonbuk National University and Biomedical Research Institute of Chonbuk National University Hospital, 20 Geonji-Ro, Geumam-dong, Deokjin-gu, Jeonju, 54907, Republic of Korea (E-mail: tcskimgh@ gmail.com).

J Thorac Cardiovasc Surg 2019;157:e367-9

$0022-5223$

Copyright (C) 2019 by The American Association for Thoracic Surgery. Published by Elsevier Inc. This is an open access article under the CC BY-NC-ND license (http://creativecommons.org/licenses/by-nc-nd/4.0/).

https://doi.org/10.1016/j.jtcvs.2019.01.004
}

- Video clip is available online.

The high takeoff left main coronary artery (LMCA) is extremely rare. ${ }^{1-3}$ The definition of high takeoff of the coronary artery remains controversial. Opinions differ as to whether this anomaly is benign or malignant, but the high takeoff of the LMCA may not be a harmless anomaly, especially when anatomic findings are associated with the acute angulation and intramural course of the high takeoff LMCA.

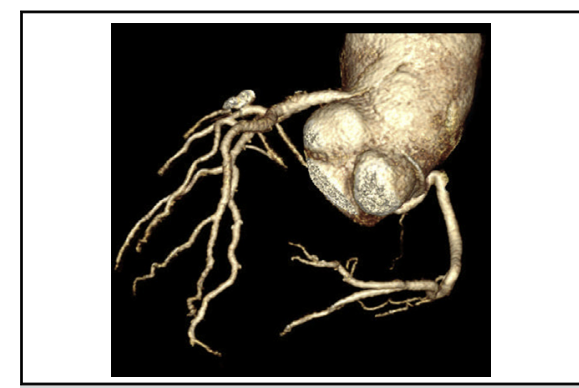

A high takeoff LMCA with severe stenosis of the proximal LMCA from acutely angled course.

\section{Central Message}

The high takeoff left main coronary artery may not be a harmless anomaly, especially when anatomic findings are associated with the acute angulation and intramural course of the high takeoff LMCA.
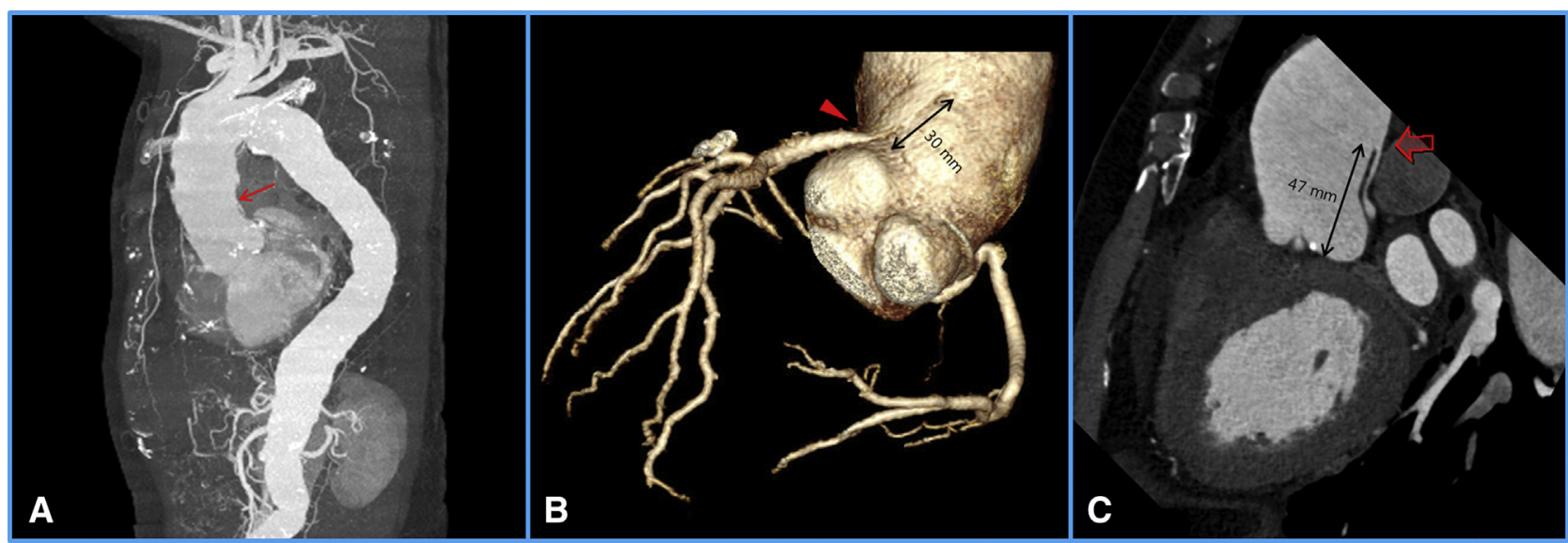

FIGURE 1. Preoperative radiologic investigations. A, Computed tomographic coronary angiogram shows marked dilatation of the ascending and arch aorta and very high take off of the left main coronary artery (arrow). B, Computed tomographic coronary angiogram showing severe stenosis of the proximal left main coronary artery because of an acute angled course (arrowhead). C, Computed tomographic coronary angiogram showing the ostium of the artery (arrow), approximately $42 \mathrm{~mm}$ above the aortic valve. 


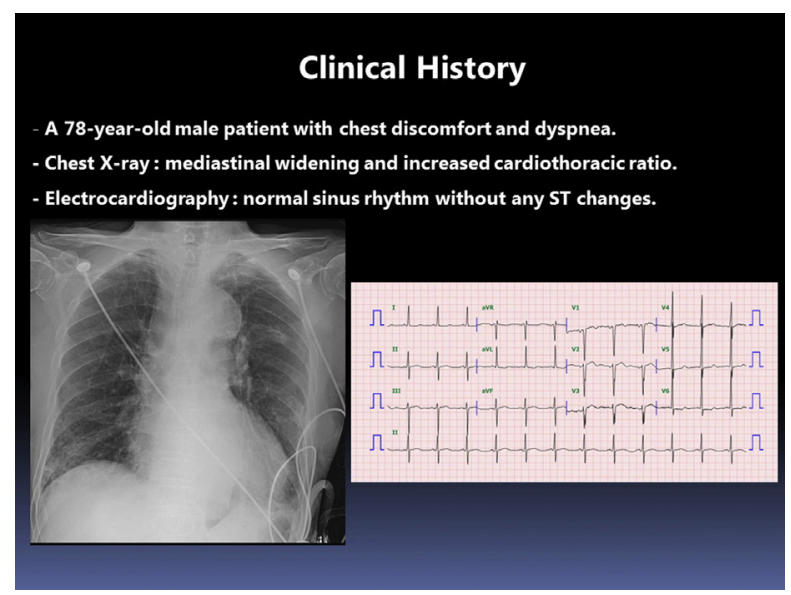

VIDEO 1. Video showing preoperative radiologic and echocardiographic findings, also including the operative images, schematic diagram, and postoperative computed tomographic coronary angiogram (CTCA). LMCA, Left main coronary artery; AoV, aortic valve. Video available at: https:// www.jtcvs.org/article/S0022-5223(19)30027-3/fulltext. acutely angled course (Figure $1, B$ and $C$, and Video 1). Transthoracic echocardiography demonstrated severe aortic regurgitation. We planned hemiarch aortic replacement and biologic Bentall procedure. After a completion of the open distal anastomosis for hemiarch aortic replacement, the aortic root dissection for preparing the Bentall procedure revealed tangential origination of the LMCA from an ostium located $30 \mathrm{~mm}$ above the sinotubular junction (Figure 2, A, and Video 1). The proximal part of the high take off of the LMCA seemed to be directed inferiorly over the left sinus of Valsalva, with the proximal membrane intramural, and the acute angulation of the LMCA was confirmed by probing (Figure 2, B, and Video 1). My team thought that the intramural course of the proximal LMCA seemed to be capable of moving to a more or less obstructive position, depending on expansion of the aortic root. To correct the potential ostial stenosis and angulation of the proximal LMCA, we decided on the unroofing procedure for the proximal LMCA. We resected the proximal membrane

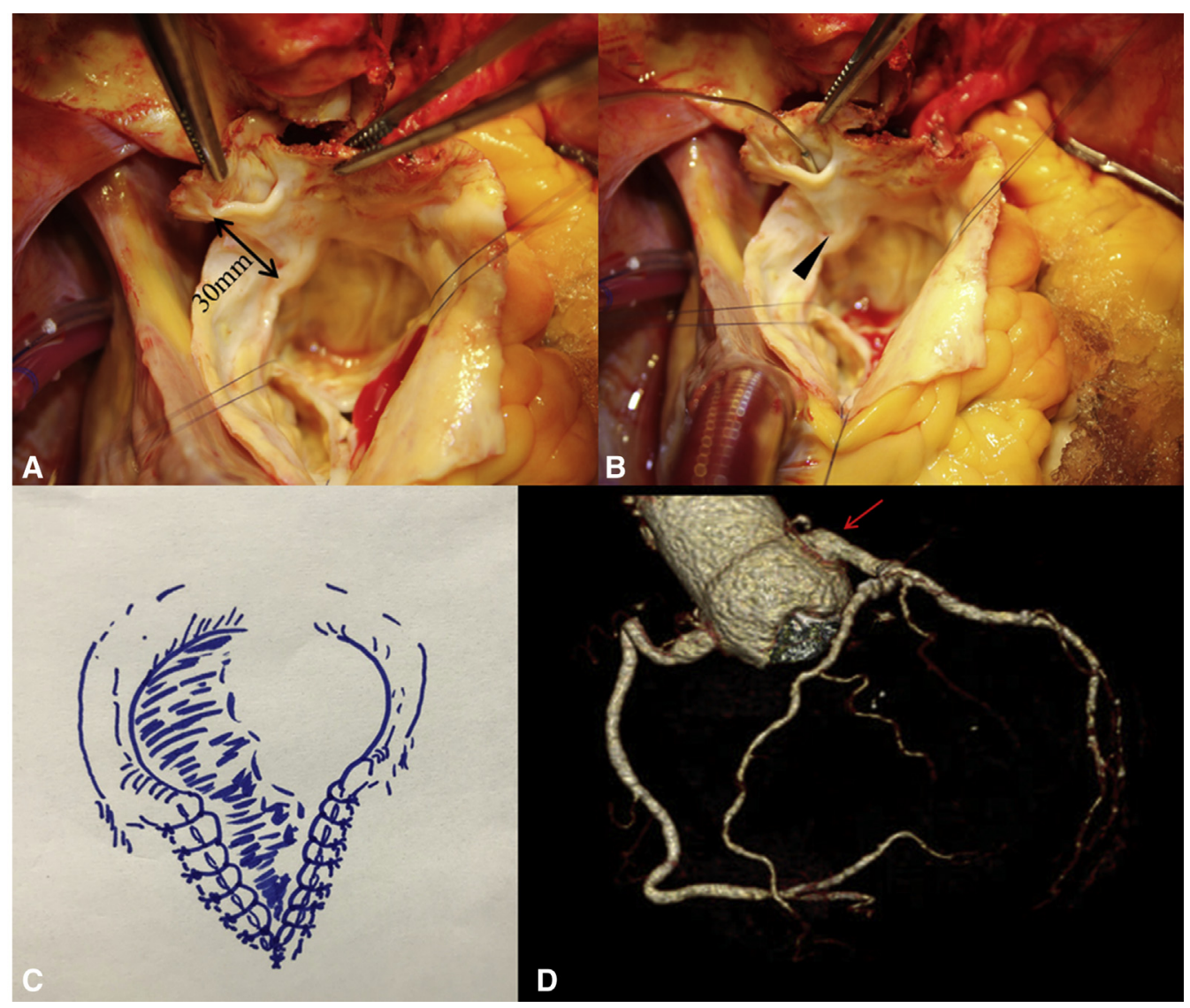

FIGURE 2. A, Tangential origination and proximal membrane of the left main coronary artery with an ostium, located $30 \mathrm{~mm}$ above the sinotubular junction. B, the acute angulation lesion (arrow head) by probing through the intramural part of the left main coronary artery. C, Schematic diagram of the unroofing procedure for the proximal left main coronary artery. D, Postoperative computed tomographic coronary angiogram showed the normal position of a patent left main coronary artery (arrow). 
and marsupialized the proximal left main intramural segment, approximating the intima of the coronary artery to the intima of the aorta (Figure 2, $C$, and Video 1). The proximally trimmed and unroofed LMCA button was reimplanted to the Valsalva graft without resection of the LMCA. The postoperative course was uneventful. The postoperative computed tomographic coronary angiogram showed a normal position of a good, patent LMCA (Figure 2, D, and Video 1). At 2 years of follow-up, there were no coronary events or prosthesis dysfunction.

\section{DISCUSSION}

The incidence of high takeoff of the LMCA is extremely low. ${ }^{1-3}$ Opinions also differ as to whether these anomalies are benign or malignant. ${ }^{4}$ This anomaly is thought to be related to myocardial ischemia and sudden death as a result of decreased coronary perfusion by displacement of the coronary ostium from the coronary sinus, ${ }^{4}$ but some still consider high takeoff to be a benign anomaly. ${ }^{1}$ In my review of the literature, I was able to find only 1 autopsy in which high takeoff of the LMCA was considered to be the main cause of ischemia or sudden death. ${ }^{5}$ As in the patient described here, we should recognize that high takeoff of the LMCA may not be a harmless anomaly when other anatomical findings are associated with an acute angulation and intramural course of the high takeoff LMCA, especially when the patient is under emotional stress. One theory explains that this patient's high takeoff LMCA had an acute angulation with an intramural course of the proximal portion because of marked cranial displacement of the ostium at the sinotubular junction as a result of the high takeoff. This theory is supported in this case by a comparison of the preoperative and postoperative images; after the unroofing procedure for the LMCA, the course of the LMCA was returned to normal along the vertical axis of the left sinus by reimplanting it at an appropriate sinus position, in contrast to its preoperative location above the sinotubular junction. The clinical significance of a high takeoff coronary ostium is clear and meaningful during cardiac surgery. When the presence of a high origin coronary artery is identified, detailed preoperative computed tomographic coronary angiography must be performed to define further the location, orientation, and course of the artery and thus avoid serious complications.

To the best of my knowledge, this is the first report to describe an intramural coursing high takeoff LMCA with acute angulation. In addition, this is the highest origin reported in a case involving the LMCA.

In conclusion, the unroofing procedure in acutely angled high takeoff LMCA is more physiological and resolves an acute angulation problem, allowing successful LMCA reimplantation for a biological Bentall procedure without resection of the intramural LMCA or torsion of the LMCA.

\section{References}

1. Yamanaka O, Hobbs RE. Coronary artery anomalies in 126,595 patients undergoing coronary angiography. Cathet Cardiovasc Diagn. 1990;21:28-40.

2. Rao R, Shwetha B. Variant high origin of both right and left coronary arteries from the ascending aortic wall. Anadolu Kardiyol Derg. 2013;13:279-80.

3. Loukas M, Andall RG, Khan AZ, Patel K, Muresian H, Spicer DE, et al. The clinical anatomy of high take-off coronary arteries. Clin Anat. 2016;29:408-19.

4. Rosenthal RL, Carrothers IA, Schussler JM. Benign or malignant anomaly? Very high takeoff of the left main coronary artery above the left coronary sinus. Tex Heart Inst J. 2012;39:538-41.

5. Naoki N, Yukiko H, Koshi K. High takeoff of the left main coronary artery at autopsy after sudden unexpected death in a male. Pathology. 2014;46:361-4. 